\title{
Finite element analysis of misaligned rotors on oil-film bearings
}

\author{
$\mathrm{S} \mathrm{SARKAR}^{1}, \mathrm{~A} \mathrm{NANDI}^{1}, \mathrm{~S} \mathrm{NEOGY}^{1}, \mathrm{~J} \mathrm{~K} \mathrm{DUTT}^{2}$ and \\ T K KUNDRA ${ }^{2}$
}

${ }^{1}$ Department of Mechanical Engineering, Jadavpur University, Kolkata 700032

${ }^{2}$ Department of Mechanical Engineering, Indian Institute of Technology,

New Delhi 110016

e-mail: arghyan@yahoo.com; anandi@mech.jdvu.ac.in

MS received 10 May 2008; revised 13 November 2009; accepted 27 November 2009

\begin{abstract}
The present work deals with a two-step nonlinear finite element analysis for misaligned multi-disk rotors on short oil-film bearings of various types (cylindrical, pocket, symmetrical three-lobed, unsymmetrical three-lobed). As a first step, the conventional parallel, angular and combined parallel and angular misalignments are modelled using Lagrange multipliers. The static equilibrium position of the journal within the bearing is determined using an iterative nonlinear static finite element analysis. The present work proposes a method for computing the displacement-dependent stiffness terms from the experimental static loaddisplacement data. Finally, the orbit of the rotor around the static equilibrium is determined using a time-integration scheme.
\end{abstract}

Keywords. Misalignment; Lagrange multiplier; journal bearing; finite elements; quadratic stiffness; time-integration scheme.

\section{Introduction}

Misalignment related malfunction of a rotor is the second most common type (Muszynska 2005) and is just next to rotor malfunction due to unbalance. Many authors commented (Muszynska 2005, Lees 2007) that a lot of work is still to be done in the field of physical understanding and modelling of misalignments. Yu \& Adams (1989) discussed about radial motion and misalignment in journal bearings and seals. By misalignment, they meant the bending (rotation) of the portion of the shaft within long bearings. They proposed a rigorous mathematical framework for linear rotor-bearing analysis with radial and rotational motion within bearings. Ding \& Krodkiewski (1993); Krodkiewski \& Ding (1993) considered an aligned rotor in misaligned multiple oil-film bearings. They solved Reynolds equation to account for the nonlinearity in the bearing. Xu \& Marangoni (1994) performed a total modelling of the motor and the driver shaft, flexible coupling and the rotor by using the method of component mode synthesis (CMS). The coupling is of universal type and allows angular 
misalignment between the driver and the driven. Since the axes of the driver and driven shafts are inclined at an angle, the torque on the driven shaft varies periodically creating angular acceleration and deceleration of the driven shaft. This gets coupled with the unbalance flexural vibration and generates higher order harmonics in unbalance response. Sekhar \& Prabhu (1995) considered standard parallel and angular misalignments at the coupling locations. They introduced a higher order finite element model to evaluate the force and moment due to misalignment. By performing a linear finite element analysis they could show the presence of the second order harmonic in response. The flexibility of the backup structures is also taken into account in the analysis. Nicolajsen demonstrated the change in the stability limit due to presence of misalignment in the arrangement of the oil-film bearings. However, nonlinearity of the bearings was not considered. Lee \& Lee (1999) derived a model of the coupling from which the force and moment at the coupling can be computed for combined misalignment. They assumed that the driver shaft is rigid compared to the flexible coupling and the driven shaft. They also used a nonlinear roller bearing model. The bearing can provide force and moment to the rotor. Experiments were carried out to validate the model. The whirling orbit deforms and collapses to straight line with increasing misalignment. Al-Hussain \& Redmond (2002) developed a set of nonlinear equations describing the motion of a system with parallel misalignment. Al-Hussain (2003) considered two rotor segments with angular misalignment. A flexible coupling was used to couple the rotor segments. Each rotor segment was mounted on two hydrodynamic bearings. The hydrodynamic bearings were modelled using standard eight-coefficient model. With the kinematic conditions considered, the kinetic and potential energy expressions were derived. The stability borderlines were computed for different values of angular misalignment and flexible coupling stiffness. Sinha et al (2004) considered that misalignment produces a constant force at the coupling. Based on this, they attempted to estimate both unbalance and misalignment from run down of different test rotors. According to Muszynska (2005) any constant radial force (or constant moment about a radial axis) on a rotor can be a cause of misalignment and can shift the normal operating point of the rotor within the bearings, which in consequence may trigger nonlinearity. This nonlinearity is the reason for presence of second and other higher order harmonics in rotor vibration. Lees $(2006,2007)$ considered two rotor segments with parallel misalignment. Idealized rigid coupling connects the rotor segments. Bolts connect the couplings to each other. While for the first rotor segment the bolts are on a circle with center at the coupling center, for the second segment the center of the pitch circle of the bolts differs from that of the coupling.

The present work proposes a two-step nonlinear finite element analysis of a rotor with parallel, angular or combined misalignment in couplings and supported by one or more oilfilm bearings. This work is an extension of Muszynska's (2005) simple two-degree of freedom rotor model to more general finite element model of shaft disc systems on multiple bearings. The present work first proposes a fairly general coupling misalignment model using Lagrange multipliers. For an integrated analysis of the driver and driven shaft, a simple coupling model is also proposed. Since, in presence of more than two bearings a rotor becomes statically indeterminate, the present work first computes the static equilibrium position of the rotor system using a nonlinear static finite element analysis. It then proposes a method to compute the quadratic nonlinear force from oil-film bearings. Using this information, the present work performs a dynamic analysis around the static equilibrium position using Newmark scheme specially adapted to tackle nonlinear forces. 


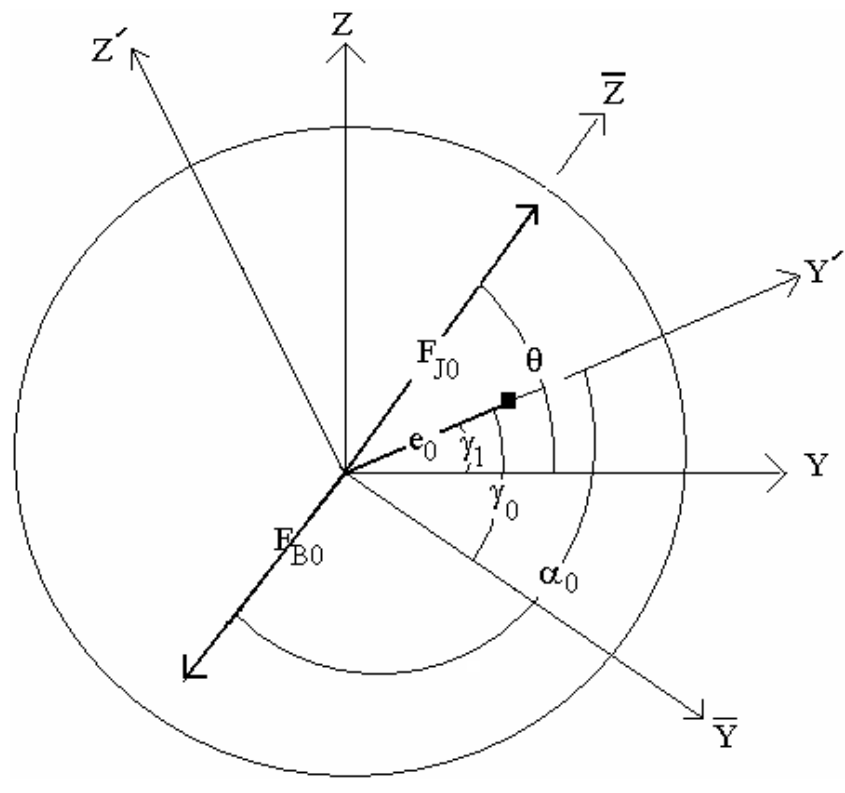

Figure 1. The coordinate systems used.

\section{Analysis}

\subsection{Coordinate systems}

The coordinate systems are shown in figure 1. For the three right-handed coordinate systems $X Y Z, X^{\prime} Y^{\prime} Z^{\prime}$ and $\bar{X} \bar{Y} \bar{Z}$, the axes $X, X^{\prime}$ and $\bar{X}$ are coincident. They are along the axis of the rotor. All the finite element equations are written with respect to the $X Y Z$ coordinate system. In the present work, the $Z$ axis points vertically upward. At each oil-film bearing location coordinate systems $X^{\prime} Y^{\prime} Z^{\prime}$ and $\bar{X} \bar{Y} \bar{Z}$ are considered. In the $X^{\prime} Y^{\prime} Z^{\prime}$ coordinate system the axis $Y^{\prime}$ points towards the static radial displacement of the journal. In the $\bar{X} \bar{Y} \bar{Z}$ coordinate system the axis $\bar{Z}$ points towards the static resultant force on the journal. The angles $\gamma_{0}, \gamma_{1}, \theta$ and $\alpha_{0}$ between different axes are indicated in figure 1 .

\subsection{Modelling of coupling misalignment in a finite element model of a rotor}

The coupling is modelled using three constituents - coupling left half $(\mathrm{CLH})$, coupling right half (CRH) and flexible part of coupling (CF). The CLH and CRH can be considered as short circular beams of large diameter so that they become almost rigid elements. The CF element is assumed to be composed of two shear springs and two rotational springs. The CF element has two nodes and just like beam elements two translation and two rotation degrees of freedom are attached with each node. Between two translation degrees of freedom in $Y$ direction at two nodes there is a shear spring. There is another shear spring between two translation degrees of freedom in $Z$ direction. Similarly, there are two rotation springs between two like pairs of rotation degrees of freedom at two nodes. No coupling between translation and rotation degrees of freedom is considered.

A schematic diagram of the coupling element is explained in figure 2. In the figure only a single plane $(X Y)$ is shown for simplicity. The two translation degrees of freedom at two nodes of CLH are $d_{n 1}$ and $d_{n 3}$. The two rotation degrees of freedom at two nodes of CLH 


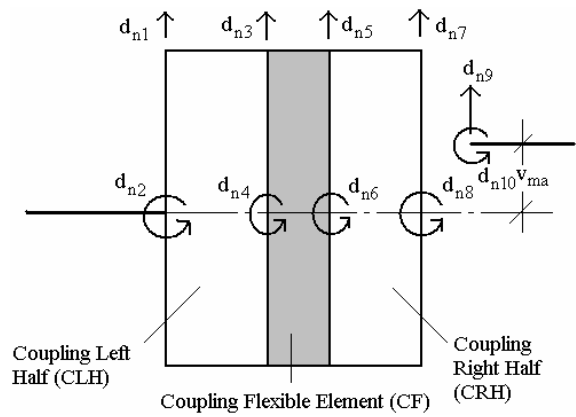

(a)

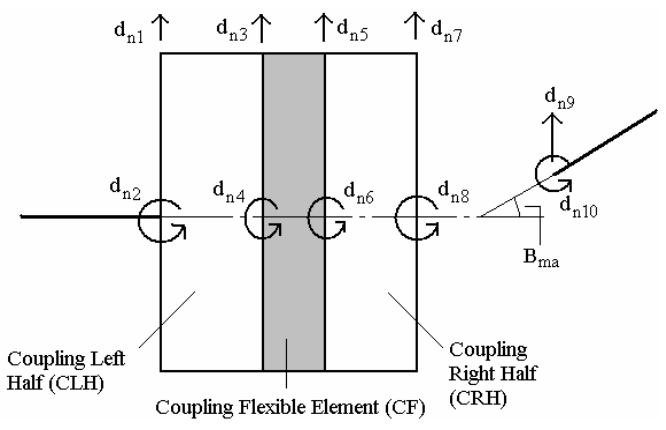

(b)

Figure 2. Schematic diagrams in two-dimension for coupling with (a) parallel misalignment and (b) angular misalignment.

are $d_{n 2}$ and $d_{n 4}$. They are actually infinitesimal rotation vectors in the $Z$ direction. Similarly, in CRH, there are four degrees of freedom $d_{n 5}, d_{n 6}, d_{n 7}$ and $d_{n 8}$. In the flexible part the two translation degrees of freedom are $d_{n 3}$ and $d_{n 5}$ and two rotation degrees of freedom are $d_{n 4}$ and $d_{n 6}$.

The shaft at the left end of the coupling is first attached to CLH. The rightmost node of this portion of the shaft share common degrees of freedom $\left(d_{n 1}\right.$ and $d_{n 2}$ in the $X Y$ plane) with left node of CLH. Then the coupling is assembled with its three constituent parts CLH, $\mathrm{CF}$ and $\mathrm{CRH}$. In case of misalignment the right node of CRH does not share all the degrees of freedom at that node with the leftmost node of the portion of the shaft located at the right side of the coupling. Let the degrees of freedom of the leftmost node of the shaft at the right of CRH be $d_{n 9}$ (translation) and $d_{n 10}$ (rotation).

For parallel misalignment in $X Y$ plane,

$$
d_{n 7}=d_{n 9}+v_{m a} .
$$

For angular misalignment in $X Y$ plane,

$$
d_{n 8}=d_{n 10}+\mathrm{B}_{m a} .
$$

The total kinetic energy of the system can be expresses as

$$
T=\frac{1}{2}\{\dot{D}\}^{T}[M]\{\dot{D}\} .
$$

The vector $\{D\}$ contains all translation and rotation degrees of the finite element model. The coupling degrees of freedom stated above are also part of the elements of the vector $\{D\}$. The matrix $[M]$ is the standard mass matrix.

Without considering the energy stored in the coupling, the total potential energy of the system is

$$
V=\frac{1}{2}\{D\}^{T}[K]\{D\}-\{D\}^{T}\{F\} .
$$

Where, the vector $\{F\}$ contains concentrated nodal force/moment along the degrees of freedom. 
Since misalignments introduce static force and moment to the system, a static analysis is performed first. The finite element equations for static analysis can be derived from the principle of minimization of potential energy. The misalignments can be considered as constraints and included in the minimization principle via Lagrange multipliers. The potential energy stored in the coupling is also now included.

$$
\begin{aligned}
V= & \frac{1}{2}\left\{D_{o}\right\}^{T}[K]\left\{D_{o}\right\}-\left\{D_{o}\right\}^{T}\left(\left\{F_{o}\right\}+\left\{F^{w t}\right\}\right)+\frac{1}{2} k_{\text {shear }}\left(d_{o n 5}-d_{o n 3}\right)^{2} \\
& +\frac{1}{2} k_{\text {rot }}\left(d_{o n 4}-d_{o n 6}\right)^{2}+\lambda_{1}\left(d_{o n 7}-d_{o n 9}-v_{m a}\right)+\lambda_{2}\left(d_{o n 8}-d_{o n 10}-\mathrm{B}_{m a}\right) .
\end{aligned}
$$

The static displacement is denoted by the symbol $\left\{D_{o}\right\}$. The symbol $d_{o j}$ stands for the entry in the $j$ th row of the static displacement vector $\left\{D_{o}\right\}$. The entry $d_{o j}$ is the static displacement for the $j$ th degree of freedom. Since in the static condition the misaligned parts of the rotor are attached using a coupling, the misalignment constraints should be applied on the static displacements. The vectors $\left\{F_{o}\right\}$ and $\left\{F^{w t}\right\}$ are the static part of the bearing force and weight of the discs respectively.

The static finite element equations can be derived using

$$
\frac{\partial V}{\partial \bar{d}_{o j}}=0
$$

Where, the term $\bar{d}_{o j}$ includes all degrees of freedom and the Lagrange multipliers. The Lagrange multipliers will now be considered as two new degrees of freedom and consequently two rows and columns will be appended to the stiffness matrix. This stiffness matrix that includes coupling stiffness and the constraint equations (incorporated via Lagrange multipliers) will be termed as $[\bar{K}]$. The force vector is also appended in the same process and denoted by $\{\bar{F}\}$.

\subsection{Derivation of displacement-dependent stiffness for short bearings from static load- deflection data}

For short cylindrical bearings the expressions for the forces on the journal in the directions $Y^{\prime}$ and $Z^{\prime}$ are as follows (Appendix A):

$$
\begin{aligned}
F_{J y^{\prime}} & =F_{\eta} f_{J Y^{\prime}}(\varepsilon, \dot{\varepsilon}, \dot{\gamma})=F_{\eta}\left\{f_{1}(\varepsilon)+\dot{\varepsilon} g_{1}(\varepsilon)+\dot{\gamma} h_{1}(\varepsilon)\right\} \\
F_{J z^{\prime}} & =F_{\eta} f_{J Z^{\prime}}(\varepsilon, \dot{\varepsilon}, \dot{\gamma})=F_{\eta}\left\{f_{2}(\varepsilon)+\dot{\varepsilon} g_{2}(\varepsilon)+\dot{\gamma} h_{2}(\varepsilon)\right\} \\
F_{\eta} & =\frac{\eta L^{3} \Omega}{2 \delta^{2}} .
\end{aligned}
$$

Where, the eccentricity ratio $\varepsilon$ is defined as $\frac{e}{\delta}$. The radial displacement of the journal is denoted by $e$. The difference between the bearing and journal radii is denoted by $\delta$. In case of a noncircular bore, a reference circle for the bearing is considered. The functions $f_{1}, f_{2}, g_{1}, g_{2}, h_{1}$ and $h_{2}$ are functions of clearance ratio $\varepsilon$ alone. These functional forms can be determined analytically. Several authors (Holmes (1960), Kramer (1990)) have presented these functional forms for short cylindrical bearing. In this work, however, approximate functional forms of $f_{1}$ and $f_{2}$ are determined from available experimental static load-deflection data. 


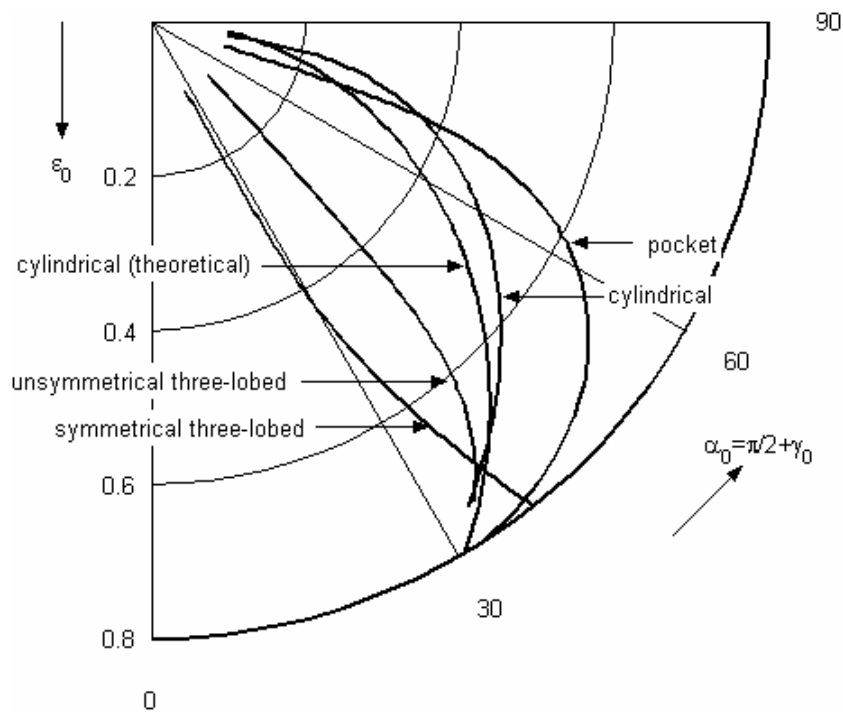

Figure 3. Experimental static load deflection curve from Glienicke along with theoretical curve for short cylindrical bearing (Kramer 1990).

Under static conditions

$$
\begin{aligned}
& F_{0_{J y^{\prime}}}=F_{\eta} f_{1}\left(\varepsilon_{0}\right), \\
& F_{0_{J z^{\prime}}}=F_{\eta} f_{2}\left(\varepsilon_{0}\right) .
\end{aligned}
$$

The eccentricity ratio under static equilibrium position is denoted by $\varepsilon_{0}$. The symbol $\gamma_{0}$ denotes the corresponding angle measured from axis $\bar{Y}$.

One can consider a case where a static reaction force $F_{J 0}$ acts on the journal is in the positive $Z$ direction. From figure 2 one can arrive at the following relation:

$$
\begin{aligned}
& f_{1}\left(\varepsilon_{0}\right) \cos \gamma_{0}-f_{2}\left(\varepsilon_{0}\right) \sin \gamma_{0}=0 . \\
& f_{1}\left(\varepsilon_{0}\right) \sin \gamma_{0}+f_{2}\left(\varepsilon_{0}\right) \cos \gamma_{0}=F_{J 0} / F_{\eta}=S^{*} .
\end{aligned}
$$

Solving for $f_{1}$ and $f_{2}$ one gets

$$
f_{1}\left(\varepsilon_{0}\right)=S^{*} \cos \gamma_{0} \quad f_{2}\left(\varepsilon_{0}\right)=S^{*} \sin \gamma_{0} .
$$

From the static load-deflection curve for a value of Sommerfeld number one finds out the corresponding values of the eccentricity ratio $\varepsilon_{0}$ and the angle $\gamma_{0}$. A table of discrete values of eccentricity ratio versus the functions $f_{1}(\varepsilon)$ and $f_{2}(\varepsilon)$ can thus be generated. An appropriate data fitting technique can then be used to approximate for the above functions. Let the approximate functions be $\hat{f}_{1}(\varepsilon)$ and $\hat{f}_{2}(\varepsilon)$ respectively. The static load-deflection curves are taken from Kramer (1990) and presented in figure 3.

In the present work, a rational function based data fitting technique has been used, where the numerator and the denominator are expressed in terms of fourth order polynomials.

$$
\begin{aligned}
& \hat{f}_{1}(\varepsilon)=\frac{\alpha_{0}+\alpha_{1} \varepsilon+\alpha_{2} \varepsilon^{2}+\alpha_{3} \varepsilon^{3}+\alpha_{4} \varepsilon^{4}}{\beta_{0}+\beta_{1} \varepsilon+\beta_{2} \varepsilon^{2}+\beta_{3} \varepsilon^{3}+\beta_{4} \varepsilon^{4}} . \\
& \hat{f}_{2}(\varepsilon)=\frac{\mu_{0}+\mu_{1} \varepsilon+\mu_{2} \varepsilon^{2}+\mu_{3} \varepsilon^{3}+\mu_{4} \varepsilon^{4}}{\nu_{0}+\nu_{1} \varepsilon+\nu_{2} \varepsilon^{2}+\nu_{3} \varepsilon^{3}+\nu_{4} \varepsilon^{4}} .
\end{aligned}
$$


Equation (7) can now be expressed in terms of the approximate functions $\hat{f}_{1}(\varepsilon)$ and $\hat{f}_{2}(\varepsilon)$

$$
\begin{aligned}
& \hat{F}_{J y^{\prime}}=F_{\eta}\left\{\hat{f}_{1}(\varepsilon)+\dot{\varepsilon} g_{1}(\varepsilon)+\dot{\gamma} h_{1}(\varepsilon)\right\} \\
& \hat{F}_{J z^{\prime}}=F_{\eta}\left\{\hat{f}_{2}(\varepsilon)+\dot{\varepsilon} g_{2}(\varepsilon)+\dot{\gamma} h_{2}(\varepsilon)\right\} .
\end{aligned}
$$

The coordinates $\varepsilon_{0}, \gamma_{0}$ represent the static equilibrium position in the polar coordinate system. The coordinates $\bar{y}_{0}, \bar{z}_{0}$ specify the same position in the $\bar{y}-\bar{z}$ Cartesian coordinate system. Now, during rotor motion, let the present position of the bearing be a little shifted from the static equilibrium position. The coordinates for the present journal center can either be represented by the variables $\varepsilon=\varepsilon_{0}+\Delta \varepsilon, \gamma=\gamma_{0}+\Delta \gamma$ or by $\bar{y}=\bar{y}_{0}+\Delta \bar{y}, \bar{z}=\bar{z}_{0}+\Delta \bar{z}$.

From equation (7) one may write

$$
\begin{aligned}
& \hat{F}_{j \bar{y}}=\hat{F}_{J y^{\prime}}(\varepsilon, \dot{\varepsilon}, \gamma) \cos \gamma-\hat{F}_{J z^{\prime}}(\varepsilon, \dot{\varepsilon}, \gamma) \sin \gamma . \\
& \hat{F}_{J \bar{z}}=\hat{F}_{J y^{\prime}}(\varepsilon, \dot{\varepsilon}, \gamma) \sin \gamma+\hat{F}_{J z^{\prime}}(\varepsilon, \dot{\varepsilon}, \gamma) \cos \gamma .
\end{aligned}
$$

From Taylor's series expansion, the stiffness matrix can be written as

$$
\left[\begin{array}{ll}
k_{\bar{y} \bar{y}} & k_{\bar{y} \bar{z}} \\
k_{\bar{z} \bar{y}} & k_{\bar{z} \bar{z}}
\end{array}\right]=-\left[\begin{array}{cc}
\frac{\partial \hat{F}_{J \bar{y}}}{\partial \bar{y}} & \frac{\partial \hat{F}_{J \bar{y}}}{\partial \bar{z}} \\
\frac{\partial \hat{F}_{J \bar{z}}}{\partial \bar{y}} & \frac{\partial \hat{F}_{J \bar{z}}}{\partial \bar{z}}
\end{array}\right] \text { Evaluated at } \varepsilon=\varepsilon_{0}, \gamma=\gamma_{0}, \dot{\varepsilon}=0, \dot{\gamma}=0 \text {. }
$$

Since the derivatives are evaluated at $\dot{\varepsilon}=0, \dot{\gamma}=0$ the functions $g_{1}, g_{2}, h_{1}, h_{2}$ will not enter the above expression.

The following standard chain-rule of differentiation is used above:

$$
\frac{\partial}{\partial \bar{y}}=\frac{\partial}{\partial \varepsilon} \frac{\partial \varepsilon}{\partial \bar{y}}+\frac{\partial}{\partial \gamma} \frac{\partial \gamma}{\partial \bar{y}} \frac{\partial}{\partial \bar{z}}=\frac{\partial}{\partial \varepsilon} \frac{\partial \varepsilon}{\partial \bar{z}}+\frac{\partial}{\partial \gamma} \frac{\partial \gamma}{\partial \bar{z}} .
$$

Where,

$$
\frac{\partial \varepsilon}{\partial \bar{y}}=\frac{1}{\delta} \cos \gamma_{0}, \quad \frac{\partial \gamma}{\partial \bar{y}}=-\frac{1}{e_{0}} \sin \gamma_{0}, \quad \frac{\partial \varepsilon}{\partial \bar{z}}=\frac{1}{\delta} \sin \gamma_{0}, \quad \frac{\partial \gamma}{\partial \bar{z}}=\frac{1}{e_{0}} \cos \gamma_{0} .
$$

Using standard coordinate transformation relation

$$
\left[\begin{array}{ll}
k_{y y} & k_{y z} \\
k_{z y} & k_{z z}
\end{array}\right]=[T]^{T}\left[\begin{array}{ll}
k_{\bar{y} \bar{y}} & k_{\bar{y} \bar{z}} \\
k_{\bar{z} \bar{y}} & k_{\bar{z} \bar{z}}
\end{array}\right][T] \text {. }
$$

Where,

$$
[T]=\left[\begin{array}{cc}
\cos \left(\frac{\pi}{2}-\theta\right) & -\sin \left(\frac{\pi}{2}-\theta\right) \\
\sin \left(\frac{\pi}{2}-\theta\right) & \cos \left(\frac{\pi}{2}-\theta\right)
\end{array}\right] .
$$

The displacement-dependent stiffnesses can be derived in a similar fashion.

$$
\begin{aligned}
& k_{\bar{y}, \bar{y} \bar{y}}=-\frac{1}{2} \frac{\partial^{2} \hat{F}_{J \bar{y}}}{\partial \bar{y}^{2}}, \quad k_{\bar{y}, \bar{y} \bar{z}}=-\frac{1}{2} \frac{\partial^{2} \hat{F}_{J \bar{y}}}{\partial \bar{y} \partial \bar{z}}, \quad k_{\bar{y}, \bar{z} \bar{z}}=-\frac{1}{2} \frac{\partial^{2} \hat{F}_{J \bar{y}}}{\partial \bar{z}^{2}} . \\
& k_{\bar{z}, \bar{y} \bar{y}}=-\frac{1}{2} \frac{\partial^{2} \hat{F}_{J \bar{z}}}{\partial \bar{y}^{2}}, \quad k_{\bar{z}, \bar{y} \bar{z}}=-\frac{1}{2} \frac{\partial^{2} \hat{F}_{J \bar{z}}}{\partial \bar{y} \partial \bar{z}}, \quad k_{\bar{z}, \bar{z} \bar{z}}=-\frac{1}{2} \frac{\partial^{2} \hat{F}_{J \bar{z}}}{\partial \bar{z}^{2}} .
\end{aligned}
$$




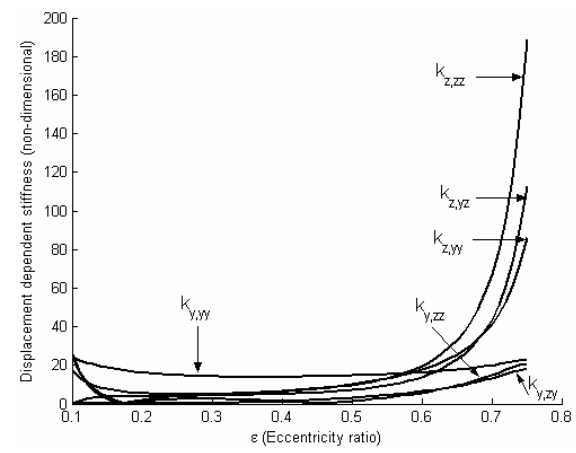

(a)

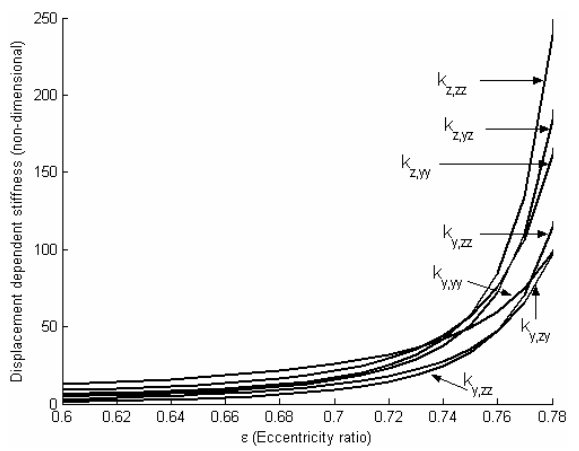

(b)

Figure 4. Plot of displacement-dependent stiffness from Taylor series expansion of approximate expressions for (a) short cylindrical bearings and (b) short pocket bearing.

The above six coefficients for short cylindrical bearing, pocket bearing, unsymmetrical and symmetrical three-lobed bearing are presented in figures 4 and 5 .

\subsection{Two-step analysis}

The motion of an unbalanced rotor in oil-film bearing is considered as a dynamic motion around a static equilibrium position. More than two bearings make a rotor statically indeterminate. In this case, under static load, an iterative scheme is employed to find out the location of the journal within the bearing.

Once the static equilibrium position due to static loads like weight and misalignment are known, one can determine the constant stiffness and displacement-dependent stiffness as described in the previous section. Then around the equilibrium position one can compute the dynamic motion of the journal. At any instant of time the total displacement of the rotor will be the algebraic sum of its static and dynamic displacements.

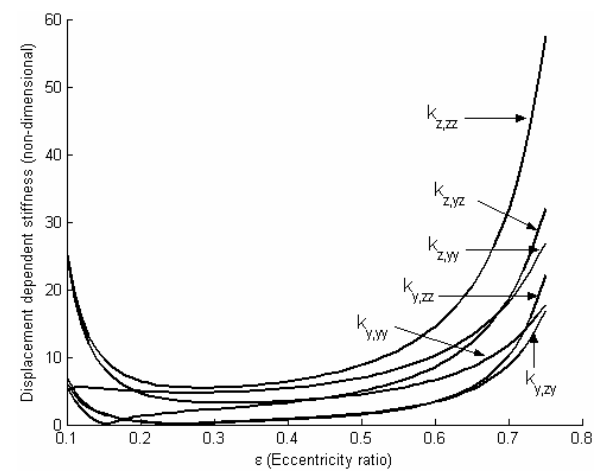

(a)

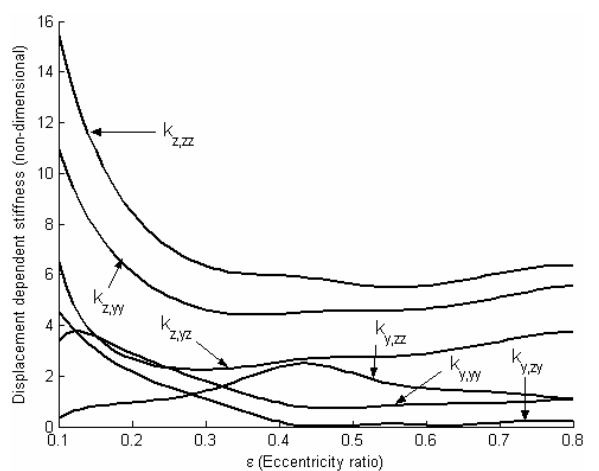

(b)

Figure 5. Plot of displacement-dependent stiffness from Taylor series expansion of approximate expressions for (a) short unsymmetrical three-lobed bearing and (b) short symmetrical three-lobed bearing. 
Therefore, the total displacement $\{D\}$ can be broken up into two parts as follows:

$$
\{D\}=\left\{D_{0}\right\}+\{\Delta D\},
$$

where, the static displacement is $\left\{D_{0}\right\}$ and the dynamic displacement is $\{\Delta D\}$.

2.4a Iterative determination of static deflection of statically indeterminate finite element rotor models: The following iterative scheme is used here for determining the static equilibrium position of the rotor.

The algorithm can be described in following steps:

(i) Form stiffness matrix $[\bar{K}]$ and right hand side force vector $\{\bar{F}\}$.

(ii) Consider large stiffness at oil-film bearing locations as a part of support conditions and have an initial guess for $\left\{\bar{D}_{0}\right\}=[\bar{K}]^{-1}\{\bar{F}\}$.

(iii) Get equilibrium force using $\left\{\bar{F}_{e q}\right\}=[\bar{K}]\left\{\bar{D}_{0}\right\}$.

(iv) Get the $Y$ and $Z$ components of forces at the oil-film bearing locations and compute the angles $\theta$.

(v) Get the $Y$ and $Z$ components of displacements at the oil-film bearing locations and compute the eccentricity ratios $\varepsilon_{0}$ angles $\gamma_{1}$.

(vi) From the values of eccentricity ratios $\varepsilon_{0}$ compute

$$
\left\{\begin{array}{c}
\hat{F}_{J y} \\
\hat{F}_{J z}
\end{array}\right\}=\left[\begin{array}{cc}
\cos \lambda_{1} & -\sin \lambda_{1} \\
\sin \lambda_{1} & \cos \lambda_{1}
\end{array}\right]\left\{\begin{array}{c}
\hat{F}_{J y^{\prime}} \\
\hat{F}_{J z^{\prime}}
\end{array}\right\}
$$

at oil-film bearing locations. For short cylindrical bearings instead of fitted function the actual theoretically obtained function may be used.

(vii) Get out of balance force vector using $\{d \bar{F}\}=\{\bar{F}\}-\left\{\bar{F}_{e q}\right\}+$ Reaction Forces on Journal.

(viii) Depending on the values of eccentricity ratios $\varepsilon_{0}$ compute stiffnesses at bearing locations and appropriately transform it in the $Y-Z$ coordinate system using equation (15).

(ix) Get the tangent stiffness matrix $\left[\bar{K}_{t}\right]=[\bar{K}]+$ Bearing stiffness.

(x) Get $\left\{d D_{0}\right\}=\left[\bar{K}_{t}\right]^{-1}\{d \bar{F}\}$.

(xi) $\left\{\bar{D}_{0}\right\}=\left\{\bar{D}_{0}\right\}+\left\{d \bar{D}_{0}\right\}$.

(xii) Go to step (iii) if convergence is not achieved.

2.4b Static equilibrium equations: As indicated by (6) the static equilibrium equations can be written as

$$
[\bar{K}]\left\{\bar{D}_{o}\right\}=\{\bar{F}\} .
$$

A way to establish this equilibrium in an iterative manner is described in the previous section 2.4a. Once the solution of equation (19a) is known one can obtain the static equilibrium equation in terms of the actual degrees of freedom $\left\{D_{o}\right\}$.

With reference to the case shown in figure 2, equation (19a) can be written as

$$
\left[\begin{array}{cc}
{[K]} & {[P]} \\
{[P]^{T}} & {[0]}
\end{array}\right]\left\{\begin{array}{c}
\left\{D_{o}\right\} \\
\left\{\begin{array}{l}
\lambda_{1} \\
\lambda_{2}
\end{array}\right\}
\end{array}\right\}=\left\{\begin{array}{c}
\left\{F_{0}\right\}+\left\{F^{w t}\right\} \\
\left\{\begin{array}{c}
v_{m a} \\
\mathrm{~B}_{m a}
\end{array}\right\}
\end{array}\right\} .
$$


The first part of equation (19b) becomes

$$
[K]\left\{D_{o}\right\}=\left\{F_{o}\right\}+\left\{F^{w t}\right\}-[P]\left\{\begin{array}{l}
\lambda_{1} \\
\lambda_{2}
\end{array}\right\} .
$$

In equation (20) from the inflated degrees freedom (due to inclusion of Lagrange multipliers) once again comes back to its original form.

Therefore, the static equilibrium equation is given by

$$
[K]\left\{D_{o}\right\}=\left\{F_{o}\right\}+\left\{F^{s t}\right\} .
$$

Where, the misalignment forces are expressed as

$$
\left\{F^{m a}\right\}=-[P]\left\{\begin{array}{c}
\lambda_{1} \\
\lambda_{2}
\end{array}\right\} .
$$

The misalignment force and the weight are combined in a single vector

$$
\left\{F^{s t}\right\}=\left\{F^{w t}\right\}+\left\{F^{m a}\right\} .
$$

The static equilibrium condition given by equation (21) is established by the iterative scheme described in the previous section 2.4 a However, the iterative scheme works on the inflated degrees of freedom.

2.4c Simplified dynamic analysis: The governing equation for dynamic analysis can be obtained as follows:

$$
[M]\{\ddot{D}\}+([C]+[G])\{\dot{D}\}+[K]\{D\}=\left\{F^{s t}\right\}+\left\{F^{\text {unb }}\right\}+\left\{F^{\text {bearing }}\right\} .
$$

The symbol $\left\{F^{s t}\right\}$ stands for static forces on the rotor due to weight and misalignment. The unbalance force vector is denoted by $\left\{F^{u n b}\right\}$. The force on the rotor from the bearing is $\left\{F^{\text {bearing }}\right\}$. The rotor is linear. The sources of nonlinearity are the forces on the rotor from the bearing.

According to equation (18) $\{D\}=\left\{D_{0}\right\}+\{\Delta D\}$, where, the static displacement is $\left\{D_{0}\right\}$ and the dynamic one is $\{\Delta D\}$. Inserting this expression for $\{D\}$ in equation (24)

$$
[M]\{\Delta \ddot{D}\}+([C]+[G])\{\Delta \dot{D}\}+[K]\left\{D_{o}+\Delta D\right\}=\left\{F^{s t}\right\}+\left\{F^{\text {unb }}\right\}+\left\{F^{\text {bearing }}\right\} .
$$

The nonlinear bearing force is expanded in a Taylor series around the static equilibrium position in a static force $\left\{F_{o}\right\}$, a linear (in displacement) force $\left\{F^{l i n}\right\}$ and a quadratic (in displacement) force $\left\{F^{n l}\right\}$.

$$
\begin{gathered}
{[M]\{\Delta \ddot{D}\}+([C]+[G])\{\Delta \dot{D}\}+[K]\left\{D_{o}+\Delta D\right\}} \\
=\left\{F^{s t}\right\}+\left\{F^{u n b}\right\}+\left\{F_{o}\right\}+\left\{F^{l i n}\right\}+\left\{F^{n l}\right\} .
\end{gathered}
$$

From static equilibrium equation (21),

$$
[K]\left\{D_{o}\right\}=\left\{F^{s t}\right\}+\left\{F_{o}\right\} .
$$

The remaining part of equation (26) is therefore,

$$
[M]\{\Delta \ddot{D}\}+([C]+[G])\{\Delta \dot{D}\}+[K]\{\Delta D\}-\left\{F^{l i n}\right\}=\left\{F^{u n b}\right\}+\left\{F^{n l}\right\} .
$$


Now, using $\left\{F^{\text {lin }}\right\}=-\left[K^{\text {bearing }}\right]\{\Delta D\}$ and $\left[K_{c}\right]=[K]+\left[K^{\text {bearing }}\right]$ one obtains

$$
[M]\{\Delta \ddot{D}\}+([C]+[G])\{\Delta \dot{D}\}+\left[K_{c}\right]\{\Delta D\}=\left\{F^{u n b}\right\}+\left\{F^{n l}\right\} .
$$

The static forces due to weight and misalignment have been taken into account in the static analysis in equation (27a) and they are not included in the equation of motion (equation 27b) for the dynamic case. The force vector $\left\{F^{n l}\right\}$ is included to account for the nonlinear forces. In the present only quadratic nonlinearity is considered.

From the plots of displacement-dependent stiffness versus eccentricity ratio (figures 4 and 5), one easily finds, that except for symmetrical three-lobed bearing (figure 5b), the displacement-dependent stiffness increases with increasing eccentricity ratio. In all the cases the value of $k_{\bar{z}, \bar{z} \bar{z}}=-\frac{1}{2} \frac{\partial^{2} \hat{F}_{J \bar{z}}}{\partial \bar{z}^{2}}$ is considerably larger than all other five such coefficients. Therefore, in this work only this single coefficient and the resulting nonlinear quadratic force are considered. After appropriate coordinate transformation one can write the following relation:

If the index $i$ denotes a node at bearing location,

$$
F_{i \bar{z}}^{n l}=-k_{\bar{z}, \bar{z} \bar{z}} \Delta u_{i \bar{z}}^{2} \text {. }
$$

The dynamic displacement at node $i$ in the direction $\bar{z}$ is represented by $\Delta u_{i \bar{z}}$

$$
\begin{aligned}
\Delta u_{i \bar{z}} & =\sin \left(\frac{\pi}{2}-\theta\right) \Delta u_{i y}+\cos \left(\frac{\pi}{2}-\theta\right) \Delta u_{i z} . \\
F_{i y}^{n l} & =F_{i \bar{z}}^{n l} \cos \theta \\
F_{i y}^{n l} & =F_{i \bar{z}}^{n l} \cos \theta .
\end{aligned}
$$

Equation (28) is integrated in time using the Newmark scheme specially adapted to handle the nonlinear force. At time step $t$ the value of the unknown displacements are to be computed. For this computation one needs to know the nonlinear force vector $\left\{F^{n l}\right\}$. But the value of this nonlinear force vector depends on the current displacements. At each time step, an iterative loop is used to accurately evaluate the quadratic force.

With reference to figure $2 \mathrm{a}$ one also notes that in the dynamic condition the degrees of freedom $\Delta d_{n 7}$ and $\Delta d_{n 9}$ move together. The same condition also applied for the rotational degrees of freedom as shown in figure $2 b$.

\section{Numerical example}

The finite element model of the rotor system used in static and dynamic analyses are shown in figure $6 \mathrm{a}$ and $6 \mathrm{~b}$ respectively. An integrated modelling of the rotor system is attempted

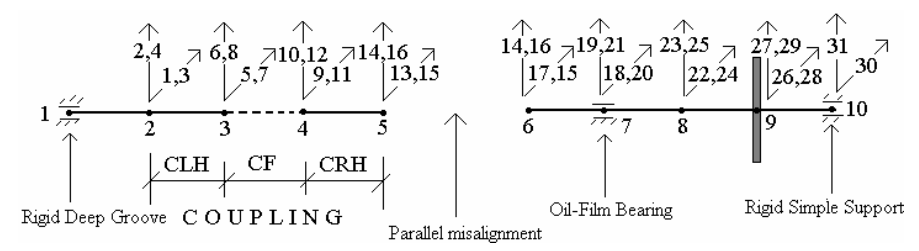

(a)

Figure 6a. The finite element model used for static analysis. 


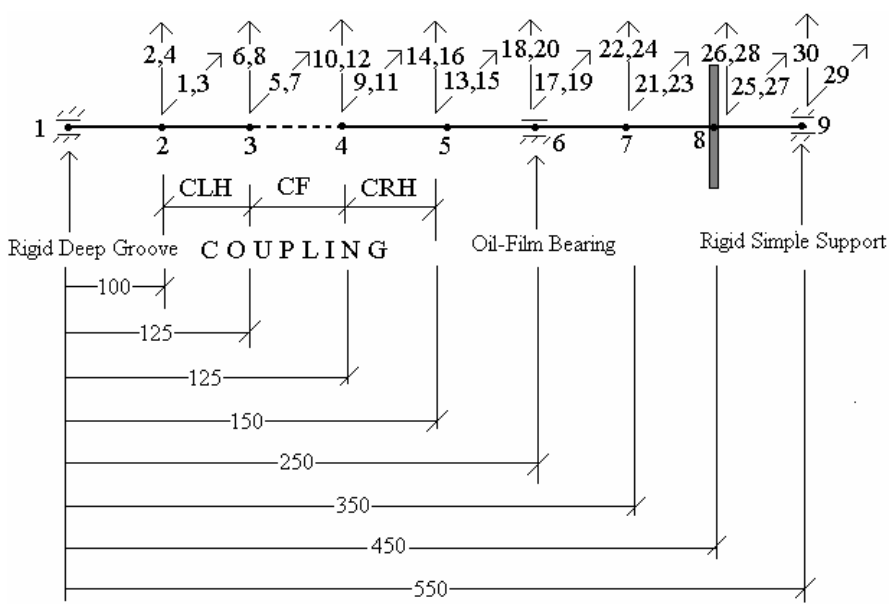

(b)

Figure 6b. The finite element model used for dynamic analysis.

considering the bearing of the motor, the coupling, one oil-film bearing and a self-aligning bearing. The oil-film bearing is of short cylindrical type. The bearing at the motor end is assumed to be of deep groove type and it does not allow translation and rotation. The selfaligning bearing is modelled as a simple support. The mild steel shaft has a diameter of $20 \mathrm{~mm}$. The dimensions along the length of the shaft are shown in figure $6 \mathrm{~b}$. The disc has a mass of $10 \mathrm{~kg}$ and radius of gyration for polar moment of inertia is $100 \mathrm{~mm}$.

The weight of the disc is a static force on the shaft. Misalignment is another source of static force and moment. In this example, only parallel misalignment in the horizontal $(Y)$ direction is considered. The location of the misalignment is close to the oil-film bearing and is shown in the figure 6 . Here, the problem is statically indeterminate. The constraint condition is $d_{0_{13}}=d_{0_{17}}+v_{m a}$.

The subscript 0 indicates static equilibrium conditions and the parallel misalignment is denoted by the symbol $v_{m a}$.

The translation and rotation stiffnesses of the coupling element are chosen high so that the coupling is almost rigid and the misalignment generates a large reaction force on the oil-film bearing.

The oil-film bearing parameters are as follows:

Length $=L=10 \mathrm{~mm}$, Clearance $=\delta=0.030 \mathrm{~mm}$, Dynamic viscosity $=\eta=0.03 \times$ $10^{-6} \mathrm{Ns} / \mathrm{mm}$. The first step in the analysis is to find out the static position of the bearing within the journal and the static displacement of the rotor system as well. Based on this analysis the constant stiffness and the displacement-dependent stiffness of the bearing will be determined and used in the subsequent nonlinear dynamic analysis. It is assumed that the rotor will have its dynamic motion around its static equilibrium position. In the static analysis the misalignment of the rotor is varied and the corresponding position of the journal is shown in figure 7 for different spin speeds of the rotor.

The following values are obtained from the static analysis for a spin speed of $500 \mathrm{rad} / \mathrm{s}$ and misalignment of $1 \mathrm{~mm}$.

Resultant static force $F_{J 0}=1312 \mathrm{~N}$, eccentricity ratio $\varepsilon_{0}=0.8$ and angle $\theta=2^{\circ}$.

A displacement-dependent stiffness of $k_{\bar{z}, \bar{z} \bar{z}}=4 \times 10^{7} \mathrm{~N} / \mathrm{mm}^{2}$ is considered. The orbit of the journal around the static equilibrium position is shown in figure 8a. The FFT of the 


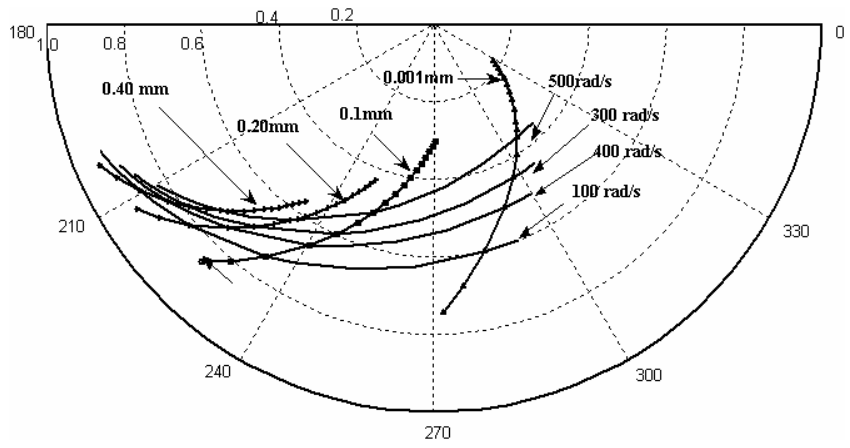

Figure 7. The static equilibrium position of journal center under constant with varying misalignment and spin speed.

displacement in the $Y$ direction indicates the presence of $2^{\text {nd }}$ and $3^{\text {rd }}$ harmonic in the response (figure $8 b$ ).

\section{Discussion}

In this work, the steady state response of a misaligned rotor subjected to unbalance excitation at a given spin speed (equation 28) is computed using numerical time-integration procedures. The response is first computed using a Newmark scheme and then the solution is verified using a Runge-Kutta method. At each time step dynamic equilibrium is established in several iterations. The time-integration starts from an initial condition. Then the transient parts slowly die down with time and the steady state solution is reached. The time step selected $\Delta t=0.00005 \mathrm{~s}$ and 40000 iterations are required before steady state conditions are obtained. In some cases of damped nonlinear vibration the steady state solution depends on initial conditions (Nayfeh \& Mook 1995). However, in the present case, the steady state solution is found to be independent of the same. Figure 8 shows the steady state solution. Figure 9 shows how from one given initial conditions the solution evolves in time.

With the advent of high speed computational facilities numerical time integration schemes have become popular in nonlinear vibration analysis. However, when one needs to compute the response of a rotor over a range of spin speed the present method has to be used repeatedly

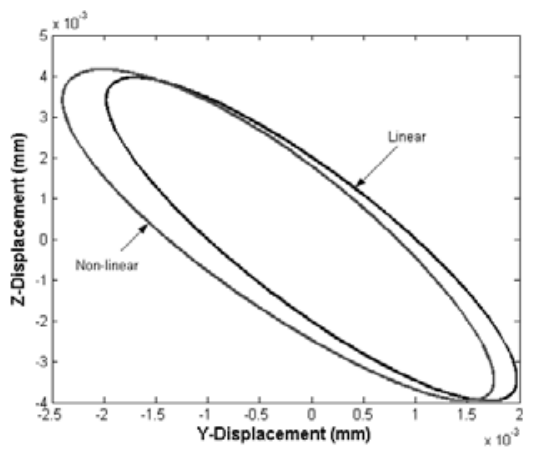

(a)

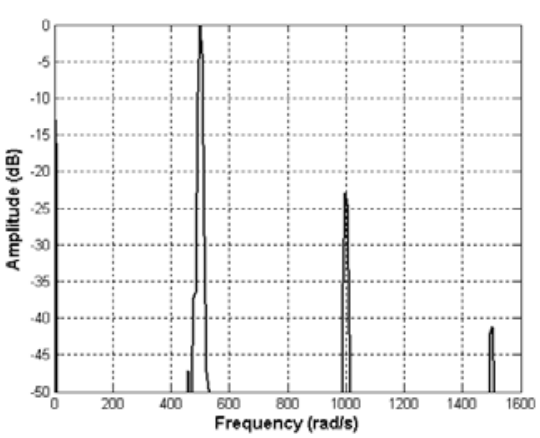

(b)

Figure 8. The (a) orbit formed by and (b) the FFT of response (in the $Y$-direction) of the journal center at a misalignment value of $1 \mathrm{~mm}$. 


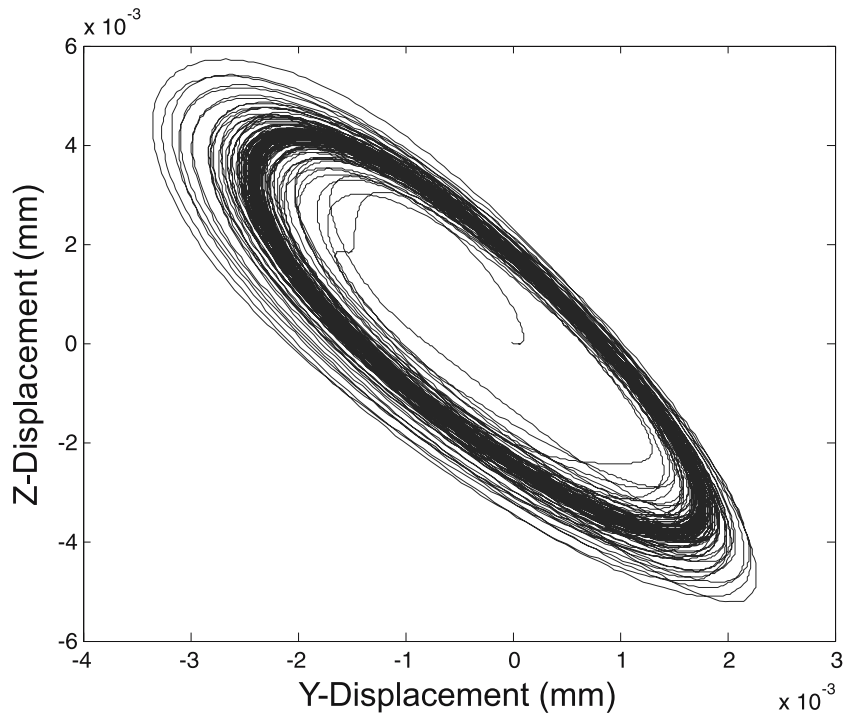

Figure 9. The evolution of the solution from initial conditions in a time-integrations scheme.

with different values of spin speed. Response computation at each spin speed requires a full time-integration. For each time-integration a large number of time steps have to be considered to reach the steady state conditions. Therefore, for studying steady state phenomena the present method is time-consuming. This is indeed a drawback of the procedure for studying steady state nonlinear phenomena like jump, bifurcation, etc. A jump in this case is sudden change in rotor orbit with a small change in spin speed.

The present work deals with multi-degree of freedom (MDOF) system with nonlinearity at bearings. The finite element model used for dynamic analysis has 30 degrees of freedom. It is difficult to apply standard perturbation techniques to such MDOF systems. However, one approach could be condensing out the linear degrees of freedom using appropriate dynamic condensation technique and then apply the standard perturbation techniques. The method of harmonic balance in combination with a continuation algorithm can be a choice (Groll \& Ewins 2001). However, the harmonic balance method inflates the number of degrees of freedom and at each frequency several iterations have to be performed. The method becomes more and more complicated with increase in number of the assumed harmonics.

\section{Conclusion}

In the present work a finite element analysis of a misaligned rotor, supported on oil-film bearing is performed. Misalignment introduces nonlinearity in vibration response. This model is, therefore, believed to be useful for development of techniques for detection of misalignment by post-processing of vibration response. Different methods based on frequency response functions can be explored for this purpose. In a rotor subjected on multiple oil-film bearings, it is also interesting to identify the bearing(s) operating in the sufficiently high nonlinear region.

\section{List of symbols}

$$
\begin{array}{ll}
p=p(X, \phi, t) & \text { Oil-film pressure } \\
X, Y, Z, Y^{\prime}, Z^{\prime}, \bar{Y}, \bar{Z}, \phi, \gamma, \theta & \text { Coordinates and angles as described in figure } 1
\end{array}
$$




$t$
$\eta$
$h=h(\phi, t)$
$h_{0}(\phi)$
$e, \gamma$
$e_{0}, \gamma_{0}$
$\{D\},\left\{D_{0}\right\},\{\Delta D\}$
$\left\{\bar{D}_{0}\right\}$
$\{\bar{F}\}$
$\bar{d}_{o j}$
$d_{j}, d_{o j}, \Delta d_{j}$
$\Delta u_{i \bar{z}}$
$F_{J y^{\prime}}, F_{J z^{\prime}}$
$\hat{F}_{J y^{\prime}}, \hat{F}_{J z^{\prime}}$
$\hat{F}_{J \bar{y}}, \hat{F}_{J \bar{z}}$
$F_{J 0}$
$\left\{F^{u n b}\right\}$
$\left\{F^{n l}\right\}$
$F_{i \bar{z}}^{n l}$
$\left\{F^{s t}\right\}$
$\left\{F^{m a}\right\}$
$\left\{F_{o}\right\}$
$\left\{F^{l i n}\right\}$
$[K],\left[K_{c}\right]$
$[C]$
$[\bar{K}]$

$[M]$

Time variable

Dynamic viscosity

Oil film thickness when the journal and the bearing centers are not coincident

Oil film thickness when the journal and the bearing centers are coincident

Polar coordinates of journal center with respect to $\bar{Y}, \bar{Z}$

Polar coordinates of journal center with respect to $\bar{Y}, \bar{Z}$ for static equilibrium

Vectors of total nodal displacements, static nodal displacements and dynamic nodal displacements respectively. $\{D\}=$ $\left\{D_{0}\right\}+\{\Delta D\}$

Inflated vector of static displacement degrees of freedom due to inclusion of Lagrange multipliers

Inflated (due to inclusion of Lagrange multiplier) static force vector of weight and static part of bearing force

$j$ th element of $\left\{\bar{D}_{0}\right\}$

Total displacement, static displacement and dynamic displacement at degree of freedom $j$ respectively

Dynamic displacement at node $i$ and direction $\bar{Z}$

Components of forces on journal in $Y^{\prime}$ and $Z^{\prime}$ directions

Components of forces on journal in $Y^{\prime}$ and $Z^{\prime}$ directions obtained after appropriate data fitting

Components of forces on journal in $\bar{Y}$ and $\bar{Z}$ directions obtained after appropriate data fitting

Resultant static force on journal

The force vector with appropriate entries for unbalance

The quadratic (in displacement) force obtained from Taylor series expansion of the bearing force

Nonlinear force at node $i$ in the direction $\bar{Z}$

Static force on the rotor due to weight of the discs

Misalignment force on the rotor

The constant part of bearing force obtained from Taylor series expansion of the bearing force

The linear (in displacement) force obtained from Taylor series expansion of the bearing force

Stiffness of the rotor without and with the bearing stiffness respectively

Stiffness of the rotor where Lagrange multipliers are included as degrees of freedom

Mass matrix of the rotor

Gyroscopic matrix of the rotor

Damping matrix of the rotor. 


\section{Appendix A}

\section{Functional form of $\boldsymbol{F}_{J y^{\prime}}$ and $\boldsymbol{F}_{J z^{\prime}}$}

For a relatively short bearing the change in pressure in the circumferential direction can be neglected in comparison with that in the axial direction. This allows one to neglect the term $\frac{\partial p}{\partial \phi}$ in the Reynolds equation. After this approximation the Reynolds equation appears as follows (Kramer 1990):

$$
\frac{\partial^{2} p}{\partial x^{2}}=\frac{6 \eta}{h^{3}}\left[\Omega \frac{\partial h_{0}}{\partial \phi}+e(\Omega-2 \dot{\gamma}) \sin (\phi-\gamma)-2 \dot{e} \cos (\phi-\gamma)\right],
$$

where

$$
h(\phi, t)=h_{0}(\phi)-e(t) \cos [\phi-\gamma(t)] .
$$

Noting that the right hand side of equation is not a function of the axial direction $x$ and using the conditions that $\frac{\partial p}{\partial x}=0$ for $x=0$ and $p=0$ for $x= \pm \frac{L}{2}$,

$p(\phi, x, t)=\frac{3 \eta}{h^{3}}\left[\Omega \frac{\partial h_{0}}{\partial \phi}+e(\Omega-2 \dot{\gamma}) \sin (\phi-\gamma)-2 \dot{e} \cos (\phi-\gamma)\right]\left(x^{2}-\frac{L^{2}}{4}\right)$.

Integrating over the length of the bearing, the pressure per unit circumferential length

$$
q(\phi, t)=F_{\eta} \frac{1}{(h / \delta)^{3}}\left[-\frac{1}{\delta} \frac{\partial h_{0}}{\partial \phi}+\varepsilon\left(\frac{2 \dot{\gamma}}{\Omega}-1\right) \sin (\phi-\gamma)+\frac{2 \dot{\varepsilon}}{\Omega} \cos (\phi-\gamma)\right],
$$

where,

$$
F_{\eta}=\frac{\eta L^{3} \Omega}{2 \delta^{2}}
$$

Force along the directions $Y^{\prime}$ and $Z^{\prime}$ can be obtained by appropriate integrations over the circumference.

Therefore,

$$
\begin{aligned}
& F_{J y^{\prime}}=F_{\eta} f_{J Y^{\prime}}(\varepsilon, \dot{\varepsilon}, \dot{\gamma})=F_{\eta}\left\{f_{1}(\varepsilon)+\dot{\varepsilon} g_{1}(\varepsilon)+\dot{\gamma} h_{1}(\varepsilon)\right\} \\
& F_{J z^{\prime}}=F_{\eta} f_{J Z^{\prime}}(\varepsilon, \dot{\varepsilon}, \dot{\gamma})=F_{\eta}\left\{f_{2}(\varepsilon)+\dot{\varepsilon} g_{2}(\varepsilon)+\dot{\gamma} h_{2}(\varepsilon)\right\} .
\end{aligned}
$$

\section{References}

Al-Hussain K M, Redmond I 2002 Dynamic response of two rotors connected by rigid mechanical coupling with parallel misalignment. J. Sound and Vibration 249(3): 483-498

Al-Hussain K M 2003 Dynamic stability of two rigid rotors connected by a flexible coupling with angular misalignment. J. Sound and Vibration 266: 217-234

Ding J, Krodkiewski J M 1990 Inclusion of static indetermination in the mathematical model for nonlinear dynamic analyses of multi-bearing rotor system. J. Sound and Vibration 164(2): 267-280

Groll, von G, Ewins D J 2001 The harmonic balance method with arc-length continuation in rotor/stator contact problems. J. Sound and Vibration 241(2): 223-233 
Holmes R 1960 The vibration of a rigid shaft on short sleeve bearings. J. Mech. Eng. Sci. 2(4):

Kramer E 1990 Dynamics of Rotors and Foundations (Heidelberg: Springer-Verlag)

Krodkiewski J M, Ding J 1993 Theory and Experiment on a method for on-site identification of configurations of multi-bearing rotor systems. J. Sound and Vibration 164(2): 281-293

Lee Y S, Lee C W 1999 Modelling and vibration analysis of misaligned rotor-ball bearing systems. J. Sound and Vibration 224(1): 17-32

Lees A W 2006 Some studies on misalignment in rigidly coupled flexible rotors. $7^{\text {th }}$ IFTOMM conference on rotor dynamics, Vienna, Austria

Lees A W 2007 Misalignment in rigidly coupled rotors. J. Sound and Vibration (in press, online version available)

Muszynska A 2005 Rotordynamics, (New York: Marcell Dekker)

Nayfeh A H, Mook D T 1995 Nonlinear oscillations (New York: John Wiley \& sons)

Sekhar A S, Prabhu B S 1995 Effects of coupling misalignment on vibrations of rotating machinery. J. Sound and Vibration 185(4): 655-671

Sinha J K, Lees A W, Friswell M I 2004 Estimating unbalance and misalignment of a flexible rotating machine from a single run-down. J. Sound and Vibration 272: 967-989

Xu M, Marangoni R D 1994 Vibration analysis a motor-flexible coupling-rotor system subject to misalignment and unbalance. J. Sound and Vibration 176(5): 663-679

Yu H, Adams M L 1989 The linear model for rotor-dynamic properties of journal bearings and seals with combined radial and misalignment motions. J. Sound and Vibration 131(3): 367-378 